\title{
Rendimiento académico, lenguaje escrito y motivación en adolescentes españoles*
}

\section{Academic Achievement, Written Language and Motivation in Spanish Teens}

Recepción: 30 Abril 2018 | Aceptación: 04 Noviembre 2019

\author{
María-José González-Valenzuela \\ Universidad de Málaga, España \\ ORCID: http://orcid.org/0000-0003-0387-0725 \\ IsAÍAs MARTín-Ruiz \\ Universidad de Málaga, España \\ ORCID: http://orcid.org/0000-0002-4696-2374
}

a Autor de correspondencia. Correo electrónico: ismar@uma.es

Para citar este artículo: González-Valenzuela, M. -J., \& Martín-Ruiz, I. (2019). Rendimiento académico, lenguaje escrito y motivación en adolescentes españoles. Universitas Psychologica, 18(4), 1-13. htt ps://doi.org/10.11144/Javeriana.upsy18-4.rale

\section{RESUMEN}

Este estudio examina la contribución individual y conjunta de la motivación, la comprensión lectora y la composición escrita en el rendimiento académico de adolescentes españoles de distintos niveles educativos. La muestra está formada por 203 sujetos, de edades comprendidas entre 12 y 14 años [1. ${ }^{\circ}$ de ESO $(M=12.4$ y $D E=1.28)$, 2. ${ }^{\circ}$ de $\operatorname{ESO}(M=13.6$ y $D E=1.02)$ y $3^{\circ}$ de $\operatorname{ESO}(M=14.2$ y $D E=1.34)$, de nivel sociocultural medio y con niveles intelectuales normales. Se evalúa el rendimiento académico, la comprensión lectora, la composición escrita y la motivación de logro. Se realizan análisis de correlación y análisis de regresión lineal. Los resultados indican que las variables conjuntamente estudiadas contribuyen a explicar un relevante porcentaje del rendimiento académico en los distintos niveles educativos. La comprensión lectora y la motivación explican entre un 44 y 64 \% y entre un 27 y $36 \%$, respectivamente, de la variabilidad el rendimiento académico en las distintas edades. Se pone de manifiesto la importancia de las variables motivacionales y lingüísticas, en concreto, de la comprensión lectora, para la mejora del rendimiento académico y de la prevención del fracaso escolar en adolescentes españoles.

\section{Palabras clave}

rendimiento académico; motivación de logro; composición escrita; comprensión lectora; adolescentes.

\footnotetext{
ABSTRACT

This study examines the joint and individual contribution of motivation, reading comprehension and written composition in the academic performance of Spanish adolescents of different educational levels. The sample consists of 203 subjects, between 12 and 14 years old [1st $\mathrm{ESO}(\mathrm{M}=12.4$ y $\mathrm{DE}=1.28)$, 2nd $\mathrm{ESO}(\mathrm{M}=13.6$ y $\mathrm{SD}=1.02)$ y $3 r d$ ESO $(M=14.2$ y $S D=1.34)]$, of medium sociocultural level and with normal intellectual levels. Academic performance, reading comprehension, written composition and achievement motivation are evaluated. Correlation analysis and linear regression analysis are performed. The results indicate that the variables studied together contribute to explain a relevant percentage of academic performance in the different educational levels. Reading comprehension and motivation
} 
explain between 44 and $64 \%$ and between 27 and 36\%, respectively, of the variability of academic performance at different ages. It highlights the importance of motivational and linguistic variables, specifically, of reading comprehension, for the improvement of academic performance and the prevention of school failure in Spanish adolescents.

Keywords

academic achievement; motivation; writing composition; reading comprehension; teens.

El estudio del rendimiento académico es un clásico objetivo en el ámbito de la psicología educativa, sobre todo por sus implicaciones en los procesos de enseñanza-aprendizaje escolar y por su relación con la eficacia y calidad del sistema educativo español (Ministerio de Educación y Ciencia [MEC], 2010). Este interés se incrementa sobre todo en las etapas educativas superiores, que es donde se encuentran mayores niveles de fracaso escolar, según los últimos informes PISA (Organización para la Cooperación y el Desarrollo Económicos [OCDE], 2019).

El rendimiento académico ha sido definido y evaluado de múltiples formas y se ha relacionado con múltiples factores, llegando estas circunstancias a dificultar su estudio (Risso, Peralbo, \& Barca, 2010). Algunos autores han definido el rendimiento académico como el conjunto de destrezas cognitivas, afectivas y sociales que el alumno adquiere de modo progresivo, relacionadas con la adquisición y utilización de los conocimientos relativos a los diferentes niveles educativos (Barca, Peralbo, Porto, \& Brenlla, 2008; Usán \& Salavera, 2018). Otros hacen referencia al logro de metas valoradas personal o socialmente en un contexto o dominio académico determinado (Wirthwein, Sparfeldt, Pinquart, Wegerer, \& Steinmayr, 2013). Por otro lado, los indicadores utilizados para evaluar el rendimiento académico también han sido diversos, destacando en frecuencia los test de aptitudes escolares (Bernardo et al., 2009; Cupani \& Lorenzo, 2010; Miñano \& Castejón, 2008; Navas, Soriano, \& Holgado, 2012), las calificaciones escolares medidas de diferentes formas o el número de asignaturas superadas durante el curso académico (Broc, 2000; Casillas et al., 2012; Miñano \& Castejón, 2008; Navas et al., 2012; Orgilés, Gómez, Piqueras, \& Espada, 2014; Plata, González, Oudhof, Valdez, \& González, 2014). La diversidad de formas de medir el rendimiento académico ha sido uno de los obstáculos con el que los investigadores se encuentran a la hora de establecer comparaciones entre los resultados encontrados en diferentes estudios. En este estudio, se ha optado por medirlo como el número de asignaturas superadas por cada estudiante en el curso académico cursado (Casillas et al., 2012; Orgilés et al., 2014; Plata et al., 2014), ya que es una medida relacionada adecuadamente con la adquisición y utilización de los conocimientos por parte del alumno, según sus profesores. Se ha descartado el uso de test de aptitudes escolares porque en la educación secundaria son escasos los instrumentos en castellano que lo miden y los que existen no hacen referencia a la adquisición de conocimientos.

En cuanto a la relación del rendimiento con otros factores, se conoce la relación determinante de las variables cognitivas y motivacionales con el rendimiento académico (Alonso Tapia \& Ruiz, 2007; Barca, Almeida, Porto, Peralbo, \& Brenlla, 2012; Mascarenhas, Almeida, \& Barca, 2005a, 2005b; Martín, Martínez-Arias, Marchesi, \& Pérez, 2008; Miñano \& Castejón, 2008). En esta línea, Barca et al. (2012) consideran que las metas académicas y las estrategias de aprendizaje y autoeficacia son determinantes relevantes del rendimiento académico, explicando ambas entre un 15 y $27 \%$ de su varianza en alumnos entre 11 y 16 años. Miñano y Castejón (2008) encuentran también, en alumnos españoles de primer curso de enseñanza secundaria, que la inteligencia, las atribuciones causales, el autoconcepto, las metas académicas y las estrategias de aprendizaje explican un $56 \%$ de la varianza del rendimiento académico, evaluado en términos de la media aritmética de las calificaciones finales. Cury, Elliot, Fonseca y Moller (2006) demuestran que las variables cognitivas son insuficientes para la explicación del rendimiento académico en alumnos de 
secundaria, y destacan el papel de las variables motivacionales, concretamente la motivación intrínseca y las metas de aprendizaje. Además, subrayan que las influencias de estas variables disminuyen conforme aumenta la adolescencia (Hustinx, Kuyper, van der Werf, \& Dijkstra, 2009). También Navas et al. (2012) encuentran en esas edades que el rendimiento académico, medido en términos de la calificación media final anual en todas las materias, es explicado por las metas de maestría y de responsabilidad social, pero no por las de interacción y autoafirmación social. Es decir, existen estudios que demuestran que ciertas variables cognitivas y motivacionales contribuyen de alguna manera a la explicación del rendimiento académico. Sin embargo, los estudios que relacionan el lenguaje escrito y el rendimiento académico son más escasos (Kern \& Friedman, 2009). Algunos estudios señalan la importancia de la intervención en edades tempranas del lenguaje escrito de manera sistemática para mejorar significativamente el rendimiento académico a lo largo de la escolaridad (González \& Delgado, 2009; González, Martín, \& Delgado, 2012; Wright, Stackhouse, \& Wood, 2008). La mayoría de estos estudios encuentran que los estudiantes más hábiles en lectura y escritura alcanzan desde edades tempranas mejores resultados en rendimiento académico, y presentan posteriormente menos problemas en el aprendizaje escolar.

Por otra parte, cabe destacar que la relación de la motivación y el lenguaje escrito también está siendo investigada. La mayoría de los estudios señalan que la motivación hacia la lectura y/o escritura explica gran porcentaje de la variabilidad de la comprensión lectora (Ho \& Guthrie, 2013; Taboada, Tonks, Wigfield, \& Guthrie, 2009) y de la composición escrita (García \& Fidalgo, 2004; González \& Martín, 2016; Martínez-Cocó, De Caso, \& García, 2009), tanto en educación primaria como secundaria. Se pone de manifiesto la importancia que tiene que los alumnos encuentren utilidad y relevancia en la tarea de escribir, tarea ardua y costosa que requiere de gran esfuerzo y tenacidad (Bruning $\&$ Horn, 2000). De la misma manera, se resalta que la motivación intrínseca y la evitación a la lectura favorecen o desfavorecen la compresión lectora, respectivamente (Guthrie, Coddington, \& Wigfield, 2009). Cabe destacar que estos trabajos relacionan la motivación con el lenguaje escrito y la consideran no como un factor general relacionado con los aprendizajes, sino como un factor específico relacionado con la tarea de leer y/o escribir.

Por tanto, se conoce la relación entre rendimiento académico y motivación (Barca et al., 2012; Cury et al., 2006; Hustinx et al., 2009; Miñano \& Castejón, 2008; Navas et al., 2012). También se ha investigado la relación entre rendimiento académico y lenguaje escrito en edades tempranas (González et al., 2012; González \& Delgado, 2009; Wright et al., 2008), así como la relación entre motivación y lenguaje escrito (García \& Fidalgo, 2004; González \& Martín, 2016; Martínez-Cocó et al., 2009; Taboada et al., 2009). Sin embargo, no son muy frecuentes los estudios que analizan la relación conjunta de la motivación de logro y la lectura y la escritura con el rendimiento académico en la adolescencia, y cuando se estudia, la motivación no se considera como un factor general relacionado con el logro académico, sino más bien como un factor específico relacionado con una tarea concreta.

Por estos motivos, el objetivo de este estudio consiste en analizar la contribución conjunta e individual de la motivación de logro y del lenguaje escrito, en términos de comprensión lectora y composición escrita, en el rendimiento académico de adolescentes españoles de distintos niveles educativos. Se espera que la contribución conjunta e individual de la motivación de logro, la comprensión lectora y la composición escrita en la explicación del rendimiento académico sea diferente en los diferentes cursos considerados de la Educación Secundaria Obligatoria. 


\section{Método}

\section{Participantes}

La muestra estuvo compuesta por 203 alumnos de Educación Secundaria Obligatoria (ESO), pertenecientes a dos centros escolares españoles elegidos al azar de una zona de nivel sociocultural medio, según los criterios establecidos por el Instituto Nacional de Estadística (INE, 2011). Los alumnos estaban distribuidos en tres grupos. El grupo 1, formado por 59 alumnos de primer curso de educación secundaria, de los cuales 38 eran niñas y 21 niños, con una edad media de 12 años y 4 meses $(D E=1.28)$; el grupo 2, formado por 67 alumnos de segundo curso de educación secundaria, de los 42 niñas y 25 niños, con una edad media de 13 años y 6 meses (DE $=1.02$ ) y el grupo 3 , formado por 77 alumnos de tercer curso de la educación secundaria, 40 niñas y 37 niños, con una edad media 14 años y 2 meses $(D E=1.34)$. Se eliminaron de la muestra un total de 39 alumnos, debido a que no hablaban castellano o presentaban déficits físicos, psíquicos o sensoriales, en función de las evaluaciones realizadas por los orientadores de los centros escolares ( $2.46 \%)$, no se disponía del consentimiento informado firmado por los padres para participar en la investigación (7.24 \%) o no realizaron las pruebas de evaluación del lenguaje escrito $(11.3 \%)$.

\section{Instrumentos}

El rendimiento académico se evaluó como el resultado del esfuerzo y del trabajo realizado por el alumno en las tareas académicas y de los conocimientos adquiridos en las distintas materias escolares, según los objetivos curriculares de cada nivel educativo. La puntuación total fue el número de asignaturas superadas por cada estudiante en el curso académico cursado (Casillas et al., 2012; Orgilés et al., 2014; Plata et al., 2014), con un rango entre 0 y 12 .

La comprensión lectora evaluó en términos de selección y organización de ideas (extracción de la idea principal de un texto y organización de las ideas principales y secundarias), conocimiento semántico (definición del significado de tres palabras relevantes del texto), conocimiento morfosintáctico (conocimiento de las características de nueve diferentes tipos de palabras y de dos diferentes tipos de oraciones) y metacognición de la comprensión lectora (conocimiento y control sobre el propio proceso de comprensión lectora) ( González \& Martín, 2016). Para la evaluación de las tres primeras variables se eligió un texto de la materia de Ciencias Sociales. La evaluación de la selección y organización de ideas se valoró entre cero y dos puntos, respectivamente; el conocimiento semántico entre cero y dos puntos cada ítem y el conocimiento morfosintáctico entre cero y tres puntos cada ítem. La puntuación total en comprensión lectora fue el sumatorio de las puntuaciones parciales obtenidas en la evaluación de cada una de estas variables. Las respuestas de cada estudiante fueron valoradas por dos examinadores, que les otorgaron diferentes puntuaciones en función de los aciertos de los participantes (González \& Martín, 2016). La fiabilidad de las valoraciones se midió en función del número de acuerdos y desacuerdos entre las valoraciones realizadas por los examinadores (93.71\%).Para la última variable, se utilizó la versión adaptada y modificada por González (1993) del Cuestionario de Consciencia Lectora (Paris, Newman, \& Jacobson, 1983), que consta de 18 ítems de opción múltiple y mide cómo el alumno evalúa la información textual, cómo planifica el proceso de comprensión lectora, cómo regula la comprensión y cuál es su conocimiento condicional. El rango de respuesta es de cero a dos puntos por ítem. La consistencia interna de este cuestionario es de 0.84. El índice de fiabilidad de las puntuaciones totales obtenidas en comprensión lectora se calculó mediante el coeficiente alfa de Cronbach $(\alpha=0.85)$.

La composición escritase evalúo en términos de progresión temática (secuenciación y coherencia de ideas en la realización de un escrito), conocimiento semántico (número de ideas coherentes que desarrolla en un 
escrito), conocimiento morfosintáctico (número de palabras utilizadas con distinto valor morfológico y concordantes en género, número y tiempo y número de oraciones concordantes utilizadas) y metacognición de la composición escrita (control y conocimiento sobre el acto de escribir y redactar un texto) (González \& Martín, 2016). Las variables progresión temática, conocimiento semántico y morfosintáctico se evaluaron a través de un escrito sobre el racismo realizado por los estudiantes, en función del número de aciertos (González \& Martín, 2016). El rango de respuesta para la progresión temática es de cero a dos puntos; para el conocimiento semántico, entre $0-15$ puntos y para el conocimiento morfosintáctico, entre 0-71 puntos. La puntuación total en composición escrita fue el sumatorio de las puntuaciones parciales obtenidas en la evaluación de cada una de estas variables. Las respuestas de cada estudiante fueron valoradas por dos examinadores. La fiabilidad de las valoraciones se midió en función del número de acuerdos y desacuerdos entre las valoraciones realizadas por los examinadores (94.01\%). La metacognición de la composición escrita se valoró mediante el Cuestionario de Evaluación de la Metacognición de la Composición Escrita(Lecuona, 1999), que consta de 23 ítems con dos opciones y mide el conocimiento sobre la planificación del proceso escritor, la búsqueda de ideas para desarrollar, la organización de las ideas a exponer, la presentación de la información escrita, la estructuración del escrito y la revisión de la composición realizada. La puntuación máxima alcanzada es el número de aciertos. La consistencia interna del cuestionario es de 0.79 . El índice de fiabilidad de las puntuaciones totales obtenidas en composición escrita se calculó mediante el coeficiente alfa de Cronbach $(\alpha=$ 0.63).

Lamotivación se calculó en función de los pensamientos relacionados con la realización de las tareas académicas y que hacen referencia a las metas perseguidas por el individuo en el afrontamiento de las mismas, a las creencias sobre la inteligencia, al modo de pensar frente a ella y a las atribuciones que el sujeto hace de sus éxitos y fracasos (Dweck \& Elliott, 1983; Weiner, 2018). Para ello, se utilizó el Cuestionario de Automensajes ([AM]; Alonso Tapia, 1995), que consta de 44 afirmaciones que los sujetos puntúan en una escala Likert de cero a cuatro, dependiendo del grado de acuerdo con el contenido del ítem. Consta de una escala de motivación positiva u orientada al aprendizaje y otra de motivación negativa. El rango de puntuaciones es de 76 puntos positivos y 106 negativos. Los índices de consistencia interna para las escalas de automensajes negativos y positivos son 0.89 y 0.75 , respectivamente (Alonso Tapia, 1995). El índice de fiabilidad de los datos obtenidos sobre motivación de logro en el estudio se calculó mediante el coeficiente alfa de Cronbach $(\alpha=0.81)$.

\section{Procedimiento}

La realización de las pruebas y la administración de los cuestionarios se llevaron a cabo de manera colectiva en cada grupo, en clase ordinaria y en horario lectivo. Se realizó una única recogida de datos, en tres sesiones para cada grupo. En primer lugar, los estudiantes contestaron al Cuestionario de Automensajes (Alonso Tapia, 1995), con el fin de evaluar la motivación de logro. En segundo lugar, durante aproximadamente una hora realizaron el escrito y se les administró el Cuestionario de Evaluación de la Metacognición de la Composición Escrita, con el fin de evaluarla. Finalmente, los alumnos, durante 45 minutos, realizaron la prueba de comprensión lectora y se les administró el Cuestionario de Consciencia Lectora (González, 1993). El número de asignaturas superadas por cada uno de los participantes durante el primer trimestre del curso académico fue proporcionado por los tutores/as de cada grupo, en función de las calificaciones otorgadas por cada profesor en cada materia.

La dirección y el consejo escolar de los centros escolares autorizaron el procedimiento de administración de las pruebas. Se informó sobre el objetivo y procedimiento del estudio y se aseguró la privacidad y protección de los 
datos personales de los participantes, a través de la firma del consentimiento informado por los padres de los participantes.

\section{Análisis de los datos}

Para analizar la relación de la comprensión lectora (CL), la composición escrita (CE) y la motivación de logro (MO) con el rendimiento académico (RA) se llevaron a cabo análisis de correlaciones ( $\mathrm{r}$ de Pearson) y análisis de regresión lineal, con el método introducir. Se ha introducido como variable dependiente el rendimiento académico y como variables independientes la composición escrita, la comprensión lectora y la motivación de logro, seleccionando los casos según el curso. Para valorar la contribución conjunta de las variables independientes al modelo de regresión se utilizaron el coeficiente de determinación (R2) y el coeficiente de determinación ajustado $\left(R \#^{2}\right)$. Para valorar la contribución de cada variable independiente sobre la varianza total de la variable dependiente se utilizó el coeficiente B.

Los supuestos de normalidad y homoceasticidad se comprobaron con la prueba Kolmogorov-Smirnov y la prueba de Levene, respectivamente. Para la evaluación de la colinealidad e independencia entre las variables se calcularon el factor de inflación de la varianza (FIV) y el índice de Tolerancia ( $\mathrm{T}$ ) con la prueba de Durbin-Watson, respectivamente.

El procesamiento y análisis estadístico de los datos se realizó con el programa Statistical Package for the Social Sciences (SPSS), versión 20.

\section{Resultados}

Tras comprobar los supuestos paramétricos de los modelos de regresión lineal en 1. de ESO, no se rechaza la hipótesis nula de normalidad $\left(Z_{C L}\right.$ $=0.88$ у $p=0.41 ; Z_{C E}=0.74$ у $p=0.62 ; y$ $\left.\mathrm{Z}_{\mathrm{MO}}=0.57 \mathrm{y} p=0.89\right)$ y de homocedasticidad entre las varianzas $\left(L_{C L}(6,42)=0.8\right.$ y $p=0.57$; $L_{C E(7,45)}=1.99$ y $p=0.07$; y $L$ MO(7,48) $=$
1.32 y $p=0.26)$. Las pruebas de colinealidad e independencia indican que no existen indicios de relación lineal entre las variables independientes y se asume la independencia entre los residuos $\left(\mathrm{T}_{\mathrm{CL}}=0.67, \mathrm{FIV}_{\mathrm{CL}}=1.48 ; \mathrm{T}_{\mathrm{CE}}=0.66, \mathrm{FIV}_{\mathrm{CE}}\right.$ $=1.5 ; \mathrm{T}_{\mathrm{MO}}=0.77$, FIV $\left._{\mathrm{MO}}=1.3 \mathrm{~B}\right)$. En2 $\stackrel{\mathrm{O}}{\mathrm{de}}$ $\mathrm{ESO}$, también se han comprobado los supuestos paramétricos de los modelos de regresión lineal y no se rechaza la hipótesis nula de normalidad $\left(Z_{C L}=0.58\right.$ y $p=0.89 ; Z_{C E}=1.21$ y $p=0.11 ; y$ $Z_{\mathrm{MO}}=0.59$ y $\left.p=0.87\right)$ ni de homocedasticidad entre las varianzas $\left(L_{C L}(10,54)=1.28\right.$ y $p=$ $0.26 ; L_{C E}(10,52)=2$ y $p=0.07$; y $L$ MO(10,54) $=$ 0.83 y $p=0.59)$. Las pruebas de colinealidad e independencia indican que no existen indicios de relación lineal entre las variables independientes y se asume la independencia entre los residuos $\left(\mathrm{T}_{\mathrm{CL}}=0.65, \mathrm{FIV}_{\mathrm{CL}}=1.52 ; \mathrm{T}_{\mathrm{CE}}=0.71, \mathrm{FIV}_{\mathrm{CE}}\right.$ $\left.=1.39 ; \mathrm{T}_{\mathrm{MO}}=0.69, \mathrm{FIV}_{\mathrm{MO}}=1.43\right)$. En el curso $3^{2}$ de ESO, se ha comprobado también los supuestos paramétricos de los modelos de regresión lineal y no se rechaza la hipótesis nula de normalidad $\left(Z_{C L}=1.02\right.$ y $p=0.24 ; Z_{C E}$ $=0.52$ у $p=0.94 ;$ у $Z_{\mathrm{MO}}=0.9$ y $\mathrm{p}=0.4$ ) ni de homocedasticidad entre las varianzas $(L$ $C L(10,53)=1.51$ у $p=0.16 ; L C E(9,49)=1.17$ у $p=0.33$; y $L \quad M O(10,64)=1.46$ у $p=0.17)$. Las pruebas de colinealidad e independencia indican que no existen indicios de relación lineal entre las variables independientes y se asume la independencia entre los residuos $\left(\mathrm{T}_{\mathrm{CL}}=0.59\right.$, $\mathrm{FIV}_{\mathrm{CL}}=1.69 ; \mathrm{T}_{\mathrm{CE}}=0.83$, FIV $_{\mathrm{CE}}=1.19 ; \mathrm{T}_{\mathrm{MO}}=$ 0.68, FIV $\left._{\mathrm{MO}}=1.46\right)$.

\section{Tabla 1}

Correlaciones entre las variables del estudio en primero curso

\begin{tabular}{crrrr}
\hline Variables & \multicolumn{1}{l}{ RA } & \multicolumn{1}{c}{ CL } & CE & MO \\
\hline RA & $1^{*}$ & & & \\
CL & $0.65^{*}$ & $1^{*}$ & & \\
CE & $0.46^{*}$ & $0.53^{*}$ & $1^{*}$ & \\
MO & $0.58^{*}$ & $0.41^{*}$ & $0.42^{*}$ & $1^{*}$ \\
\hline
\end{tabular}

Nota.RA (Rendimiento Académico); CL (Comprensión Lectora); CE (Composición Escrita); MO (Motivación). ${ }^{*} p<0.001$ 
Por otra parte, las correlaciones entre las distintas variables del estudio en cada curso son significativas. En el primer curso, se observa que todas las correlaciones son estadísticamente significativas, siendo mayores los coeficientes entre rendimiento académico y comprensión lectora, seguido de rendimiento académico y motivación, y de la comprensión lectora y composición escrita (Tabla 1). En segundo curso, todas las variables correlacionan significativamente, siendo mayores los coeficientes entre rendimiento académico y comprensión lectora, seguido de los de rendimiento académico y motivación, comprensión lectora y motivación, así como comprensión lectora y composición escrita (Tabla 2). En tercer curso, todas las variables correlacionan significativamente, salvo entre composición escrita y motivación. Las correlaciones son mayores entre rendimiento académico y comprensión lectora, comprensión lectora y motivación, rendimiento académico y motivación, así como comprensión lectora y composición escrita (Tabla 3).

Tabla 2

Correlaciones entre las variables del estudio en segundo curso

\begin{tabular}{crrrr}
\hline Variables & \multicolumn{1}{c}{ RA } & \multicolumn{1}{c}{ CL } & CE & MO \\
\hline RA & $1^{*}$ & & & \\
CL & $0.6^{*}$ & $1^{*}$ & & \\
CE & $0.39^{*}$ & $0.48^{*}$ & $1^{*}$ & \\
MO & $0.52^{*}$ & $0.5^{*}$ & $0.43^{*}$ & $1^{*}$ \\
\hline
\end{tabular}

Nota. RA (Rendimiento Académico); CL (Comprensión Lectora); CE (Composición Escrita); MO (Motivación). * p $<0.001$

Para el primer curso, los resultados del análisis de regresión muestran que la correlación entre comprensión lectora, composición escrita y motivación con el rendimiento académico es alta $[r(49)=0.73, p=0.001]$ (Tabla 4). El ajuste del modelo es significativo $[F(3,45)=18.03$, $p<0.01, f^{2}=0.32$ ] e incluye las variables comprensión lectora $[t(49)=3.82, p<0.01]$ y motivación de logro $[t(49)=3.15, p<0.01$. El coeficiente de determinación ajustado indica que $52 \%$ de la varianza del rendimiento académico es explicada por el modelo de regresión ( $R$ $\#^{2}$ ajustado $\left.=0.52\right)$. La comprensión lectora contribuye en un $46 \%$ y la motivación en un 36 $\%$ a la explicación del rendimiento académico.

Tabla 3

Correlaciones entre las variables del estudio en tercer curso

\begin{tabular}{crrrr}
\hline Variables & \multicolumn{1}{c}{ RA } & \multicolumn{1}{c}{ CL } & CE & MO \\
\hline RA & $1^{*}$ & & & \\
CL & $0.76^{*}$ & $1^{*}$ & & \\
CE & $0.39^{*}$ & $0.4^{*}$ & $1^{*}$ & \\
MO & $0.51^{*}$ & $0.55^{*}$ & 0.17 & $1^{*}$ \\
\hline
\end{tabular}

Nota. RA (Rendimiento Académico); CL (Comprensión Lectora); CE (Composición Escrita); MO (Motivación). *p $<0.001$

Para el segundo curso, los resultados del análisis de regresión muestran que la correlación entre comprensión lectora, composición escrita y motivación con el rendimiento académico es alta $[r(62)=0.66, p=0.001]$ (Tabla 4). El ajuste del modelo es significativo $[F(3,58)=14.97$, $p<0.01, f^{2}=0.32$ ] e incluye las variables comprensión lectora $[t(62)=3.61, p<0.01]$ y la motivación de logro $[t(62)=2.32, p<0.01$. El coeficiente de determinación ajustado indica que $41 \%$ de la varianza del rendimiento académico es explicada por el modelo de regresión ( $R$ $\#^{2}$ ajustado $\left.=0.41\right)$. La comprensión lectora contribuye en un $44 \%$ y la motivación en un 27 $\%$ a la explicación del rendimiento académico.

\section{Tabla 4}

Análisis de regresión del rendimiento académico en todos los grupos

\begin{tabular}{|c|c|c|c|c|c|c|c|c|c|}
\hline Curso & $R$ & $R^{2}$ & $\begin{array}{c}R^{2} \\
\text { Ajustada }\end{array}$ & $F$ & Variables & B & SE & $t$ & $p$ \\
\hline \multirow{3}{*}{$1 .^{\circ}$} & \multirow{3}{*}{0.73} & \multirow{3}{*}{0.54} & \multirow{3}{*}{0.52} & \multirow{3}{*}{$18.03^{*}$} & CL & 0.46 & 0.02 & 3.82 & 0.01 \\
\hline & & & & & $\mathrm{CE}$ & 0.06 & 0.03 & 0.53 & 0.59 \\
\hline & & & & & $\mathrm{MO}$ & 0.3 & 0.02 & 3.15 & 0.01 \\
\hline \multirow{3}{*}{$2 .^{\circ}$} & \multirow{3}{*}{0.66} & \multirow{3}{*}{0.43} & \multirow{3}{*}{0.41} & \multirow{3}{*}{$14.97^{*}$} & CL & 0.44 & 0.02 & 3.62 & 0.01 \\
\hline & & & & & $\mathrm{CE}$ & 0.06 & 0.04 & 0.54 & 0.58 \\
\hline & & & & & MO & 0.27 & 0.02 & 2.33 & 0.02 \\
\hline \multirow{3}{*}{$3 .^{\circ}$} & \multirow{3}{*}{0.77} & \multirow{3}{*}{0.6} & \multirow{3}{*}{0.58} & \multirow{3}{*}{$24.85^{*}$} & $\mathrm{CL}$ & 0.64 & 0.03 & 5.52 & 0.01 \\
\hline & & & & & $\mathrm{CE}$ & 0.11 & 0.02 & 1.14 & 0.25 \\
\hline & & & & & $\mathrm{MO}$ & 0.12 & 0.02 & 1.18 & 0.24 \\
\hline
\end{tabular}

Nota.RA (Rendimiento Académico); CL (Comprensión Lectora); CE (Composición Escrita); MO (Motivación). ${ }^{*} p<=0.01$

Para el tercer curso, los resultados del análisis de regresión muestran que la correlación entre 
comprensión lectora, composición escrita y motivación con el rendimiento académico es alta $[r(53)=0.77, p=0.001]$ (Tabla 4). El ajuste del modelo es significativo $[F(3,49)=24.85$, $\left.p<0.01, f^{2}=0.32\right]$ e incluye solo la variable comprensión lectora $[t(53)=5.52, p<0.01]$. El coeficiente de determinación ajustado indica que $58 \%$ de la varianza del rendimiento académico es explicada por el modelo de regresión ( $R$ \# $^{2}$ ajustado $\left.=0.58\right)$. La comprensión lectora contribuye en un $64 \%$ a la explicación del rendimiento académico.

\section{Discusión}

El objetivo de este estudio consistió en analizar la contribución conjunta e individual de la motivación de logro y del lenguaje escrito, en términos de comprensión lectora y composición escrita, en el rendimiento académico de adolescentes españoles de distintos niveles educativos.

Los resultados obtenidos indican que el lenguaje escrito y la motivación explican conjuntamente un porcentaje relevante $y$ diferente de la variabilidad del rendimiento académico en todos los cursos, y que su contribución individual varía según el curso. La contribución conjunta de todas las variables consideradas oscila entre el 41 y el $58 \%$. La comprensión lectora y la motivación explican entre un 44 y $64 \%$ y entre un 27 y $36 \%$, respectivamente, de la variabilidad el rendimiento académico, según el curso. En concreto, en primero y segundo curso, la comprensión lectora y la motivación son las variables que contribuyen a la explicación del rendimiento académico, mientras que, en el tercer curso, solo la comprensión lectora contribuye a su explicación. En todos los cursos, la comprensión lectora es la que explica más variabilidad del rendimiento académico. La contribución de la composición escrita no es significativa en ningún curso. Estos resultados van en la línea de los encontrados por Risso et al. (2010). Estos autores demostraron que el rendimiento académico global (agrupado en niveles según los percentiles obtenidos) es explicado de diferente forma según el curso. En segundo curso de educación secundaria, el rendimiento académico fue explicado por la valoración familiar del estudio, el autoconcepto académico general, la comprensión lectora, la orientación/motivación al esfuerzo personal y el autoconcepto físico. En el cuarto curso, el rendimiento académico fue explicado por la valoración familiar del estudio, la comprensión lectora, la orientación/motivación al esfuerzo personal, el motivo profundo \# estrategia profunda\# y la valoración positiva de la capacidad. En ambos cursos, la comprensión lectora resultó estar entre las tres primeras variables que predicen el rendimiento académico, después del autoconcepto académico y la valoración familiar del estudio y antes que la motivación.

Por otra parte, nuestros resultados coinciden también con los obtenidos en otras investigaciones, donde se defiende el papel determinante de la motivación en el rendimiento académico (Alonso Tapia \& Ruiz, 2007; Barca et al., 2012; Cury et al., 2006; Dweck, 2018; Miñano \& Castejón, 2008; Navas et al., 2012; Panadero \& Alonso-Tapia, 2014). La mayoría de estos trabajos han encontrado a estas edades porcentajes de predicción similares a los encontrados en nuestro estudio, a pesar de que el rendimiento académico se ha medido de manera diferente en cada uno de ellos y se han considerado otras variables potencialmente predictoras diferentes junto con la motivación. En este sentido, se puede afirmar que la motivación intrínseca orientada a metas de aprendizaje en la adolescencia favorecería la mejora del rendimiento académico; es decir, fomentaría la constancia y el esfuerzo en la consecución de las tareas escolares y la consideración de las mismas como un reto para el aprendizaje, y no como una amenaza u obstáculo en las que deben probar sus conocimientos. Sin embargo, los resultados indican que la relación de la motivación con el rendimiento académico es diferente en cada nivel educativo, disminuyendo su influencia con la edad. A este respecto, algunos estudios demuestran que la relación de 
la motivación con el rendimiento académico no es progresiva, sino que fluctúa según los niveles educativos y la edad de los sujetos, y que parece disminuir a medida que aumenta la experiencia educativa (Alonso-Tapia, Panadero, \& Díaz, 2014; Hustinx et al., 2009; Laidra, Pullmann, \& Allik, 2007). Esto podría ser debido a que el desarrollo de la motivación lectora en educación secundaria no fue progresivo y, por tanto, su influencia tampoco. Sería necesario realizar estudios longitudinales que demostrasen la evolución de la motivación de logro en estas edades con el fin de poder confirmar que estos resultados son debidos a cómo se comporta esta variable en estos niveles educativos.

Al igual que otras investigaciones desde edades tempranas (González \& Delgado, 2009; Wright et al., 2008), los resultados del presente estudio defienden también la relación de la lectoescritura con el rendimiento académico. Se ha encontrado que la comprensión lectora es un fuerte predictor del rendimiento académico en la adolescencia, incrementándose su relación con la edad. Es decir, cabe esperar que cuanto más hábiles en comprensión lectora sean los estudiantes mejores resultados alcancen en las distintas tareas académicas y menos problemas presenten en el aprendizaje escolar (González et al., 2012). Estos resultados coinciden con otros estudios que examinan conjuntamente la contribución de la comprensión lectora con otras variables cognitivas y motivacionales en la adolescencia, destacando su relevancia entre las otras (Risso et al., 2010). Llama la atención que la comprensión lectora sea la variable que más explica el rendimiento académico y que su influencia sea creciente. Esto puede ser debido a las exigencias y demandas de las tareas que los estudiantes tienen que realizar en estos niveles educativos, donde el acto de comprender lo que leen se convierte en una pieza clave para la adquisición de conocimientos. Sería interesante analizar en estudios futuros la relación entre la compresión lectora y otras variables cognitivas, como la motivación de logro, con el fin de establecer las influencias entre ellas y si esto pudiera influir en la contribución en la explicación del rendimiento académico.
Por otra parte, en nuestro estudio no se ha encontrado relación entre la composición escrita y el rendimiento académico a estas edades, a pesar de la relación de ambas variables con la motivación, como indican algunos estudios (García \& Fidalgo, 2004; González \& Martín, 2016; Martínez-Cocó et al., 2009). La manera de medir la composición escrita en este estudio puede determinar los resultados obtenidos, ya que esta variable está constituida por medidas de diferente dificultad, y pueden no discriminar todas igual. Sería recomendable que en estudios futuros se analice la influencia de la composición escrita en el rendimiento académico, teniendo en cuenta cada una de sus dimensiones por separado y que se analice esta relación en profundidad dado que no son muy abundantes los trabajos en esta línea.

Por tanto, los resultados encontrados en este estudio sugieren la importancia de variables motivacionales y lingüísticas en el rendimiento académico en la adolescencia. Se destaca el carácter predictivo de la comprensión lectora por encima del de la composición escrita e incluso del de la motivación de logro en todos los niveles educativos considerados. Mientras que la motivación parece disminuir su influencia a lo largo de estas edades, la comprensión lectora aumenta su carácter predictivo. Cabe esperar que cuanto mejor sea la comprensión lectora de los estudiantes mejor será su rendimiento académico, mientras que cuanto peor sea la comprensión lectora peor rendimiento académico tendrán. Para la adquisición de nuevos aprendizajes escolares, la comprensión lectora es un vehículo importante que garantiza su éxito y evita las dificultades de aprendizaje desde edades tempranas. A pesar de que los resultados van en la misma línea de los obtenidos en algunos estudios, deben considerarse con cautela, debido a que no se conoce el tamaño de la población a la que pertenece la muestra de estudio, no pudiéndose conocer su grado de representatividad.

En estudios futuros, sería interesante analizar el rendimiento académico de los adolescentes españoles que presentan problemas de comprensión lectora en estas edades, 
con el fin de profundizar en el carácter explicativo de esta variable con respecto a otras, como es la motivación de logro, en estas poblaciones. También sería importante la creación de pruebas psicométricas que midan en el contexto educativo el rendimiento académico en educación secundaria en términos de adquisición de conocimientos, con el fin de poder establecer comparaciones de los resultados obtenidos en otros estudios y en este, dónde se ha evaluado el rendimiento académico en términos de asignaturas superadas en el curso escolar.

Finalmente, los resultados encontrados tienen importantes implicaciones educativas de cara a la mejora del rendimiento académico en la adolescencia de alumnos españoles. Parece que el lenguaje escrito, y en concreto de la comprensión lectora, junto con la motivación de logro, influye en el rendimiento académico. Se pone de manifiesto la importancia de ambas variables en educación secundaria, como vehículo para mejorar el rendimiento académico y disminuir el fracaso escolar (González et al., 2012; Slavin, 2004).

\section{Referencias}

Alonso Tapia, J. (1995). Orientación educativa. Teoría, evaluación e intervención. Madrid: Síntesis.

Alonso Tapia, J., Panadero, E., \& Díaz, M. A. (2014). Development and validity of the Emotion and Motivation Self-Regulation Questionnaire (EMSR-Q). The Spanish Journal of Psychology, 17,E 55. https://doi.or g/10.1017/sjp.2014.41

Alonso Tapia, J., \& Ruiz, M. (2007). Learningrelated motives and the perception of the motivational quality of the learning environment. Psicothema, 19(4), 603-609. Recuperado de http://www.psicothema.co $\mathrm{m} /$ psicothema.asp?id $=3404$

Barca, A., Almeida, L. S., Porto, A. M., Peralbo, M., \& Brenlla. J. C. (2012). Motivación escolar y rendimiento: impacto de las metas académicas, de estrategias de aprendizaje y autoeficacia. Anales de Psicologia, 28(3),
848-859. https://doi.org/10.6018/analesps. 28.3.156101

Barca, A., Peralbo, M., Porto, A., \& Brenlla, J. C. (2008). Contextos multiculturales, enfoques de aprendizaje y rendimiento académico en el alumnado de Educación Secundaria. Revista Iberoamericana de Educación, 46, 193-226. https://doi.org/10. $35362 /$ rie460723

Bernardo, A. B., Núñez, J. C., González-Pienda, J. A., Rosario, P., Álvarez, L., GonzálezCastro, P., ... Rodríguez, C. (2009). Estilos intelectuales y rendimiento académico: una perspectiva evolutiva. Psicothema, 21(4), 555-561. Recuperado de http://www.psicot hema.com/psicothema.asp? $\mathrm{id}=3670$

Broc, M. A. (2000). Autoconcepto, autoestima y

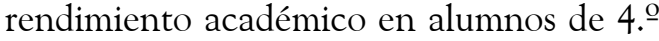
de la ESO. Implicaciones psicopedagógicas en la orientación y tutoría. Revista de Investigación Educativa, 18(1), 119-146. Recuperado de https:/digitum.um.es/digitu $\mathrm{m} /$ handle/10201/45186

Bruning, R., \& Horn, C. (2000). Developing motivation to write. Educational Psychologist, 35(1), 25-37. https://doi.org/10 $.1207 /$ S15326985EP3501_4

Casillas, A., Robbins, S., Allen, J., Kuo, Y., Hanson, M. A., \& Schmeiser, C. (2012). Predicting early academic failure in high school from prior academic achievement, psychosocial characteristics, and behavior. Journal of Educational Psychology, 104(2), 407-420. https://doi.org/10.1037/a0027180

Cupani, M., \& Lorenzo, J. (2010). Evaluación de un modelo social-cognitivo del rendimiento en matemática en una población de preadolescentes argentinos. Infancia y Aprendizaje, 33(1), 63-74. https://doi.org/1 $0.1174 / 021037010790317216$

Cury, F., Elliot, A., Fonseca, D., \& Moller, A. (2006). The social-cognitive model of achievement motivation and the $2 \times 2$ achievement goal framework. Journal of Personality and Social Psychology, 90, 666679. https://doi.org/10.1037/0022-3514.90. 4.666 
Dweck, C. S. (2018). Reflections on the legacy of attribution theory. Motivation Science, 4(1),17-18. https://doi.org/10.1037/mot00 00095

Dweck, C. S., \& Elliott, D. S. (1983). Achievement motivation. En P. H. Mussen \& E. M. Hetherington (Eds.), Handbook of child psychology (Vol. 4, pp. 326-341). Nueva York: Wiley.

García, J. N., \& Fidalgo, R. (2004). El papel del autoconocimiento de los procesos psicológicos de la escritura en la calidad de las composiciones escritas. Revista de Psicología General y Aplicada, 5(3), 281-298. Recuperado de https://dialnet.unirioja.es/se rvlet/articulo?codigo $=1318519$

González, M. C. (1993). Análisis metacognitivo de la comprensión lectora: un programa de evaluación e intervención de alumnos de enseñanza primaria. Madrid: Universidad Complutense de Madrid.

González, M. J., \& Delgado, M. (2009). Rendimiento académico y enseñanzaaprendizaje de la lectoescritura en Educación Infantil y Primaria: un estudio longitudinal. Infancia y Aprendizaje, 32(3), 265-276. Recuperado de https://dialnet.uni rioja.es/servlet/articulo? codigo $=3033225$

González, M. J., \& Martín, I. (2016). Motivación de logro y lenguaje escrito en adolescentes españoles. Anales de Psicología, 32(2), 466-473. https://doi.org/10.6018/analesps. 32.2.201561

González, M. J., Martín I., \& Delgado, M. (2012). Teaching literacy and decreased risk of learning disabilities. Revista de Psicodidáctica, 17(2), 253-268. Recuperado de https://www.redalyc.org/pdf/175/175231 28002.pdf

Guthrie, J. T., Coddington, C. S., \& Wigfield, A. (2009). Profiles of motivation for reading among African American and Caucasian students. Journal of Literature Research, 41(3),317-353. https://doi.org/10.1080/108 62960903129196

Ho, A. N., \& Guthrie, J. T. (2013).Patterns of association among multiple motivations and aspects of achievement in reading.
Reading Psychology, 34(2), 101-147. https:// doi.org/10.1080/02702711.2011.596255

Hustinx, P. W. J., Kuyper, H., van der Werf, M. P. C., \& Dijkstra, P. (2009). Achievement motivation revisited: New longitudinal data to demonstrate its predictive power. Educational Psychology, 29, 561-582. https:/ /doi.org/10.1080/01443410903132128

Instituto Nacional de Estadística. (2011). Información estadística. https://www.ine.es/p rodyser/pubweb/Censos2011_25Mapas/25 mapas_flash/files/assets/basic-html/page-1. html\#

Kern, M. L., \& Friedman, H. S. (2009). Early educational milestones as predictors of lifelong academic achievement, midlife adjustment and longevity. Journal of Applied Developmental Psychology, 30, 419-430. http s://doi.org/10.1016/j.appdev.2008.12.025

Laidra, K., Pullmann, H., \& Allik, J. (2007). Personality and intelligence as predictors of academic achievement: A cross-sectional study from elementary to secondary school. Personality and Individual Differences, 42, 441-451. https://doi.org/10.1016/j.paid.200 6.08 .001

Lecuona, M. P. (Dir.). (1999). Pensar para escribir: un programa de enseñanza para la composición escrita. Madrid: Ministerio de Educación y Ciencia, Centro de Investigación y Documentación Educativa.

Martín, E., Martínez-Arias, R., Marchesi, A., \& Pérez, E. M. (2008). Variables that predict academic achievement in the Spanish Compulsory Secondary Educational System: A longitudinal, multilevel analysis. The Spanish Journal of Psychology, 211(2), 400-413. Recuperado de https://revistas.ucm.es/index.php/SJOP/ article/view/SJOP0808220400A/28713

Martínez-Cocó, B., De Caso, A. M., \& García, J. N. (2009). Composición escrita y motivación: una perspectiva de desarrollo. Aula Abierta, 37(1), 129-140. Recuperado de http://redined.mecd.gob.es/xmlui/handl e/11162/4880

Mascarenhas, S., Almeida, L. S., \& Barca, A. (2005a). Atribuições causais e rendimento 
escolar: Impacto das habilitaçoes escolares dos pais e do género dos alunos. Revista Portuguesa de Educação, 18, 77-91. Recuperado de https://www.redalyc.org/pdf /374/37418105.pdf

Mascarenhas, S., Almeida, L. S., \& Barca, A. (2005b). Estilos atribucionais e rendimento académico: Um estudo com estudantes brasileiros do ensino médio. Revista GalegoPortuguesa de Psicoloxía e Educación, 10, 221-228. Recuperado de https://dialnet.uni rioja.es/servlet/articulo? codigo $=1292323$

Ministerio de Educación y Ciencia. (2010). PISA 2009. Programa para la Evaluación Internacional de los Alumnos OCDE. Informe español. Madrid: Ministerio de Educación, Instituto de Evaluación. Recuperado de htt p://www.educacion.es/dctm/ministerio/hor izontales/prensa/notas/2010/20101207-pisa 2009-informe-espanol.pdf?documentld $=0$ $901 \mathrm{e} 72 \mathrm{~b} 806 \mathrm{ea} 35 \mathrm{a}$

Miñano, P., \& Castejón, J. L. (2008). Capacidad predictiva de las variables cognitivomotivacionales sobre el rendimiento académico. Revista Electrónica de Motivación y Emoción, 11(28). Recuperado de http://reme.uji.es/articulos/numero28/ar ticle4/article4.pdf

Navas, L., Soriano, J. A., \& Holgado, F. B. (2012). Metas múltiples y rendimiento académico: perfiles motivacionales en educación física. Revista Mexicana de Psicología, 29(1), 33-39. Recuperado de https://www.redalyc.org/art iculo.oa?id=243030189003

Organización para la Cooperación y el Desarrollo Económicos. (2019). PISA 2018. Assessment and analytical framework. París: OCDE Publishing. https://doi.org/10. 1787/b25efab8-en

Orgilés, M., Gómez, M., Piqueras, J. A., \& Espada, J. P. (2014). Relación entre el rendimiento escolar y la sintomatología depresiva en niños españoles. Electronic Journal of Research in Educational Psychology, 12 (2),447-460. https://doi.org/10.14204/ejr ep.33.14009

Panadero, E., \& Alonso-Tapia, J. (2014). Teorías de autorregulación educativa: una comparación y reflexión teórica. Psicología Educativa. Revista de los Psicólogos de la Educación, 20(1), 11-22. https://doi.org/10. 1016/j.pse.2014.05.002

Paris, S. G., Newman, R. S., \& Jacbos, J. E. (1983). Becoming a strategic reader. Contemporary Educational Psychology, 8, 293-316. https://doi.org/10.1016/0361-476 X(83)90018-8

Plata, L. D., González, N. I., Oudhof, H., Valdez, J. L., \& González, S. (2014). Factores psicológicos asociados con el rendimiento escolar en estudiantes de educación básica. Revista Intercontinental de Psicología y Educación, 16(2), 131-149. https ://doi.org/10.1016/0361-476X(83)90018-8

Risso, A., Peralbo, M., \& Barca, A. (2010). Cambios en las variables predictoras del rendimiento escolar en enseñanza secundaria. Psicothema, 22(4), 790-796. Recuperado de http://www.psicothema.co $\mathrm{m} /$ psicothema.asp? $\mathrm{id}=3803$

Slavin, R. E. (2004). Built to last: Long-term maintenance of success for all. Remedial and Special Education, 25(1),61-66. https://doi.o $\mathrm{rg} / 10.1177 / 07419325040250010701$

Taboada, A., Tonks, S. M., Wigfield, A., \& Guthrie, J. T. (2009). Effects of motivational and cognitive variables on reading comprehension. Reading and Writing, 22, 85-106. https://doi.org/10.1007/s11145-00 8-9133-y

Usán, P., \& Salavera, C. (2018). Motivación escolar, inteligencia emocional y rendimiento académico en estudiantes de Educación Secundaria Obligatoria. Actualidades en Psicología, 32 (125), 95-112. https://doi.org/10.15517/ap.v32i125.32123

Wright J. A., Stackhouse J., \& Wood, J. (2008). Promoting language and literacy skills in the early years: Lessons from interdisciplinary teaching and learning. Child Language Teaching and Therapy, 24(2), 155-171. https://doi.org/10.1177/02656590 07090292

Weiner, B. (2018). The legacy of an attribution approach to motivation and emotion: A no- 
crisis zone. Motivation Science, 4(1), 4-14. h ttps://doi.org/10.1037/mot0000082

Wirthwein, L., Sparfeldt, J. R., Pinquart, M., Wegerer, J., \& Steinmayr, R. (2013). Achievement goals and academic achievement: A closer look at moderating factors. Educational Research Review, 10, 66-89. https://doi.org/10.1016/j.edurev.201 3.07 .001

\section{Notas}

* Artículo de investigación. 